CRYSTALLOGRAPHIC COMMUNICATIONS

ISSN 2056-9890

\section{Crystal structure of tetraisobutylthiuram disulfide}

\author{
Patricia R. Fontenot, ${ }^{\mathrm{a}}$ Bo Wang, ${ }^{\mathrm{a}}$ Yueli Chen ${ }^{\mathrm{b}}$ and James P. Donahue ${ }^{\mathrm{a} *}$
}

Received 29 August 2017

Accepted 17 October 2017

Edited by A. J. Lough, University of Toronto, Canada

Keywords: crystal structure; tetrathiuram disulfide; dithiocarbamate; precursor; weak $\mathrm{S}$...H-C interaction.

CCDC reference: 1580550

Supporting information: this article has supporting information at journals.iucr.org/e

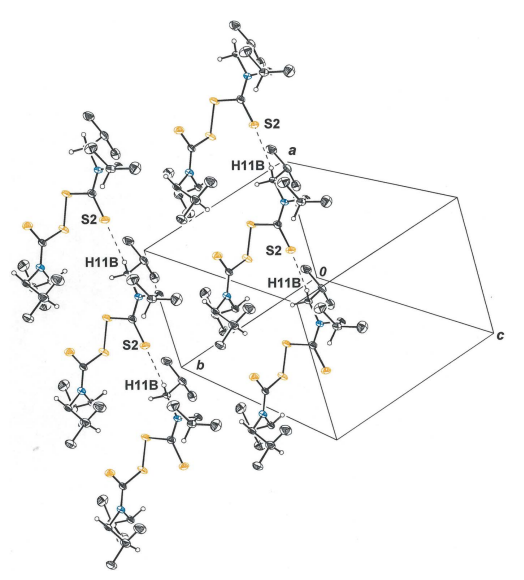

OPEN $\odot$ ACCESS

\section{Chemical context}

$N, N, N^{\prime}, N^{\prime}$-Tetraalkylthioperoxydicarbonic diamides, commonly called tetrathiuram disulfides, comprise a class of organosulfur compounds with applications that are both diverse and long-standing. Tetramethylthiuram disulfide, known by the commercial name thiram, is broadly useful both as a fungicide (Sharma et al., 2003) and as a repellent against animals that feed upon seedling trees (Radwan, 1969). In industry, thiram and related tetraalkylthiuram disulfides find application as vulcanizing agents in the production of synthetic rubber (Datta \& Ingham, 2001; Ignatz-Hoover \& To, 2016). Tetraethylthiuram disulfide, under the trade name disulfiram, is used for the treatment of chronic alcoholism because of its inhibitory effect upon liver alcohol dehydrogenase (Mutschler et al., 2016). More recently, it has received scrutiny for its ability to sensitize cancer cells to radiotherapy and to the effects of anticancer drugs (Jiao et al., 2016) as well as for its bactericidal action against drug-resistant Mycobacterium tuberculosis (Horita et al., 2012). Tetraalkylthiuram disulfides function both as chelating ligands themselves (Chieh, 1977; Chieh, 1978; Thirumaran et al., 2000; Saravanan et al., 2005; Prakasam et al., 2009) and as precursors to dithiocarbamate ligands, which are used in the coordination chemistry of both the transition metals (Hogarth, 2005) and main group elements (Heard, 2005).

In the course of some studies of diisobutyldithiocarbamate coordination complexes of molybdenum, we have noted a report describing an ${ }^{1} \mathrm{H}$ NMR spectrum of $\left[\mathrm{Ni}\left(\mathrm{S}_{2} \mathrm{CN}^{i} \mathrm{Bu}_{2}\right)_{2}\right]$ that was more complex than anticipated, even considering the hindered rotation about the ${ }^{-} \mathrm{S}_{2}-\mathrm{CN}^{i} \mathrm{Bu}_{2}$ bond (Raston \& White, 1976). This complexity was attributed to intraligand $\mathrm{S} \cdot \mathrm{H}$ interactions involving the tertiary hydrogen of the 
isobutyl group. Although the room temperature ${ }^{1} \mathrm{H}$ NMR spectrum of $N, N, N^{\prime}, N^{\prime}$ - tetrakis(2-methylpropyl)thioperoxydicarbonic diamide (tetraisobutylthiuram disulfide) itself does not show evidence of such intramolecular interaction, several recent studies of tetrathiuram disulfides have suggested such interactions in the crystalline state (Raya et al., 2005; Srinivasan et al., 2012; Nath et al., 2016). This possibility of similar weak interaction(s) in the crystal structure of tetraisobutylthiuram disulfide has motivated a determination of its structure by X-ray diffraction, reported herein.

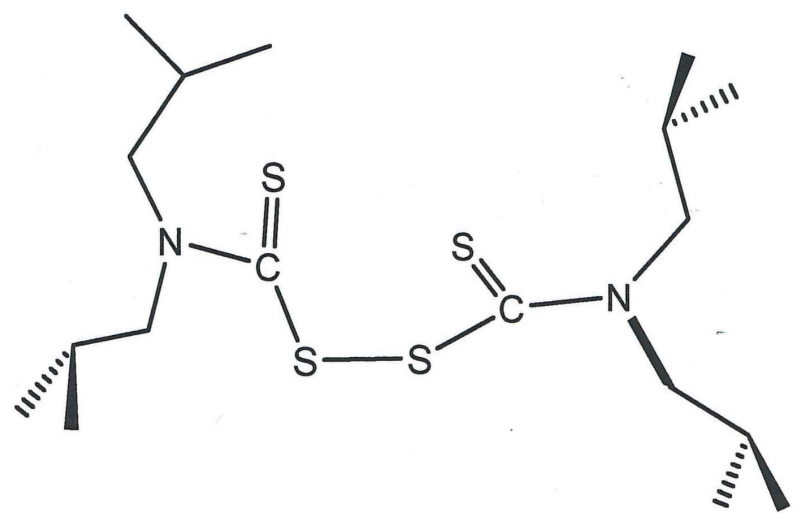

\section{Structural commentary}

Tetraisobutylthiuram disulfide crystallizes upon a general position in $P \overline{1}$ but has pseudo- $C_{2}$ symmetry across the disulfide bond, strict $C_{2}$ symmetry being disrupted by conformational differences among the pendant isobutyl groups (Fig. 1a). Despite the lack of strict $C_{2}$ symmetry, tetraisobutylthiuram disulfide is nevertheless chiral. The image in Fig. $1 a$ presents the molecule with a left-handed configuration to the core $\mathrm{H}_{2} \mathrm{CNC}(\mathrm{S}) \mathrm{S}-\mathrm{SC}(\mathrm{S}) \mathrm{NCH}_{2-}$ portion. If Fig. $1 a$ were to be viewed from above, along the pseudo $C_{2}$ axis that bisects the $\mathrm{S} 3-\mathrm{S} 4$ bond, the $\mathrm{C} 1-\mathrm{S} 1$ and $\mathrm{C} 2-\mathrm{S} 2$ thione bonds would project forward and backward, respectively, from the plane of the paper and thereby define a left-handed propeller. The right-handed counterpart is necessarily the other occupant of the unit cell, as required by the racemic space group. Among the structurally characterized thiuram disulfides, crystallographically imposed $C_{2}$ symmetry is also common (Fig. 3).

The S3-S4 bond length is 1.9931 (10) $\AA$, while the thione $\mathrm{C}=\mathrm{S}$ bonds are essentially identical at $1.642(3)$ and 1.643 (3) $\AA$. The $\mathrm{C} 1-\mathrm{S} 3-\mathrm{S} 4-\mathrm{C} 2$ torsion angle, $\tau$, is $-85.81(2)^{\circ}$ and, as is typical of tetrathiuram disulfides, very similar in magnitude to the angle of $85.91(5)^{\circ}$ between the mean planes defined by the $\mathrm{S}_{2} \mathrm{CN}$ fragments, $\theta$.

Multiple intramolecular S. $\cdot \mathrm{H}-\mathrm{C}$ contacts that are shorter than, or close to, the $2.92 \AA$ sum of the van der Waals radii (Rowland \& Taylor, 1996) for sulfur and hydrogen are calculated for the structure of tetraisobutylthiuram disulfide. Each of the four sulfur atoms on the molecule is a participant in such a close contact, as illustrated in Fig. $1 b$ and shown in Table 1. Although weak individually, particularly since these $D-\mathrm{H} \cdots A$ angles are closer to $90^{\circ}$ than to $180^{\circ}$ (Table 1 ), these interactions may act cooperatively with packing forces to decide the specific molecular conformation that is adopted. Weak intermolecular S $\cdots \mathrm{H}-\mathrm{C}$ contacts are also calculated for molecules that stack along the $a$ axis of the cell (Fig. 2). While angles for these contacts are larger $\left(145.6,159.5^{\circ}\right)$, the $D \cdots A$ separations are longer $[3.834(3), 3.810(3) \AA]$. The geometric parameters for both these intramolecular and intermolecular S . . C-H contacts fall within the range defined as consistent with a weak $D-\mathrm{H} \cdots \mathrm{A}$ interaction (Desiraju \& Steiner, 1999). These features of the molecular packing in the crystal structure of tetraisobutylthiuram disulfide suggest that the crystal structures of coordination complexes with the diisobutyldithiocarbamate ligand be considered for similar $\mathrm{S} \cdots \mathrm{H}-\mathrm{C}$ contacts and, importantly, that variable temperature ${ }^{1} \mathrm{H}$ NMR spectroscopy be used to assess the importance of any such interactions in solution.

\section{Supramolecular features}

Molecules of tetraisobutylthiuram disulfide are linked by $\mathrm{C}-$ $\mathrm{H}$...S hydrogen bonds (Table 1) to form linear chains

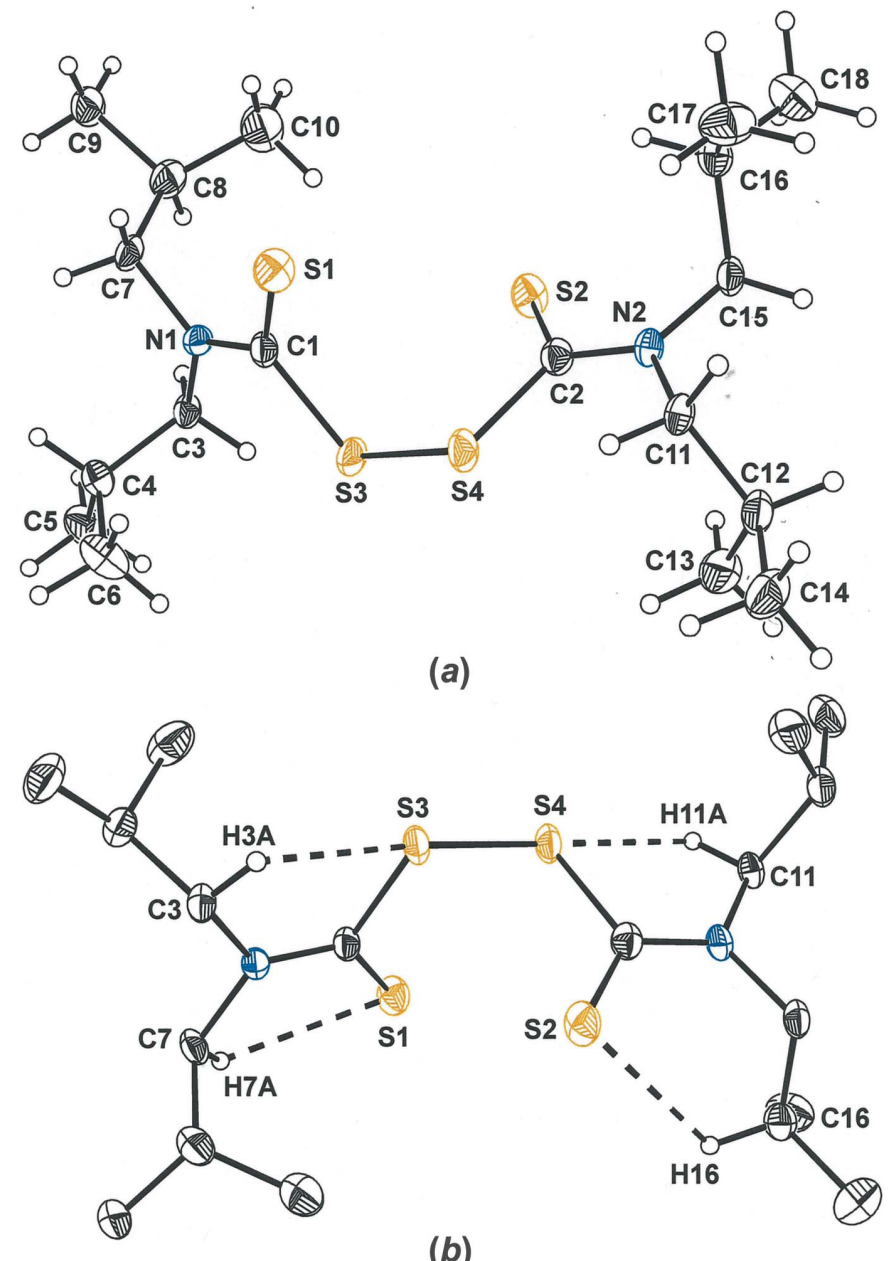

Figure 1

(a) Displacement ellipsoid plot $(50 \%)$ of tetraisobutylthiuram disulfide with complete labeling for the non-H atoms. (b) Displacement ellipsoid plot (50\% probability) of tetraisobutylthiuram disulfide illustrating close intramolecular $\mathrm{S} \cdots \mathrm{H}-\mathrm{C}$ contacts (dashed lines). 
Table 1

Hydrogen-bond geometry $\left(\AA,^{\circ}\right)$.

\begin{tabular}{|c|c|c|c|c|}
\hline$D-\mathrm{H} \cdots A$ & $D-\mathrm{H}$ & $\mathrm{H} \cdots A$ & $D \cdots A$ & $D-\mathrm{H} \cdots A$ \\
\hline $\mathrm{C} 3-\mathrm{H} 3 A \cdots \mathrm{S} 3$ & 0.99 & 2.34 & 2.907 (3) & 115 \\
\hline $\mathrm{C} 7-\mathrm{H} 7 A \cdots \mathrm{S} 1$ & 0.99 & 2.60 & $3.084(3)$ & 110 \\
\hline $\mathrm{C} 8-\mathrm{H} 8 \cdots \mathrm{S} 1^{\mathrm{i}}$ & 1.00 & 2.97 & $3.834(3)$ & 146 \\
\hline $\mathrm{C} 11-\mathrm{H} 11 A \cdots \mathrm{S} 4$ & 0.99 & 2.33 & $2.896(3)$ & 115 \\
\hline $\mathrm{C} 11-\mathrm{H} 11 B \cdots \mathrm{S} 2^{\mathrm{ii}}$ & 0.99 & 2.87 & $3.810(3)$ & 160 \\
\hline $\mathrm{C} 16-\mathrm{H} 16 \cdots \mathrm{S} 2$ & 1.00 & 2.91 & $3.473(3)$ & 117 \\
\hline
\end{tabular}

Symmetry codes: (i) $x+1, y, z$; (ii) $x-1, y, z$.

directed along the $a$ axis of the cell, and parallel chains then align within the $a b$ plane to form sheets (Fig. 2). Because the molecules within a single sheet are related, one from another, only by translations along $a$ or $b$, they all have the same optical configuration. The sheets in the $a b$ plane then stack along the $c$ axis of the cell. The cell's inversion center resides within the center of the cell and relates molecules from neighboring sheets. Consequently, the sheets alternate in the handedness of the molecules from which they are comprised.

\section{Database survey}

Values for $\tau$ and $\theta$ for structures in the Cambridge Structural Database (Web CSD v1.1.1; Groom et al., 2016) were determined using Mercury (Macrae et al., 2008). These structures are: METHUS (Marøy, 1965), METHUS01 (Ymén, 1983), METHUS02 (Wang et al., 1986), METHUS03 (Wang \& Liao, 1989), METHUS04 (Wang \& Liao, 1989), ETHUSS (Karle et al., 1967) ETHUSS01 (Wang et al., 1986), ETHUSS02 (Wang \& Liao, 1989), ETHUSS03 (Wang \& Liao, 1989), ETHUSS04 (Shi \& Wang, 1992), ETHUSS05 (Hu, 2000), HIQJUM (Jian et al., 1999), HIQJUM01 (Yu \& Wang, 2003), JECYAZ (Kumar et al., 1990), TIBFEQ (Zhai et al., 2007), ZEMPUC (Hall \& Tiekink, 1995), KAZHEA (Karim et al., 2012), NELTUT (Fun et al., 2001), XEBJOF (Ajibade et al., 2012), JAXPOO (Raya et al., 2005), CAPLEK (Williams et al., 1983), CAPLEK01 (Ymén, 1983), CAPLEK02 (Yamin et al., 1996), CAPLEK03 (Bai et al., 2010), RISNEN (Quan et al., 2008), ULOXIC (Bodige \& Watson, 2003), PIPTHS (Dix \& Rae, 1973), PIPTHS01 (Shi \& Wang, 1992), EWESUW (Nath et al., 2016), BOMPAU (Rout et al., 1982), VOHFIH (Polyakova \& Starikova, 1990), VOHFIH01 (Ivanov et al., 2003), PECWOL (Uludağ et al., 2013), ZIJLOV (Srinivasan et al., 2012) and MEMFUG (Sączewski et al., 2006).

The $\mathrm{C}-\mathrm{S}-\mathrm{S}-\mathrm{C}$ torsion angle $(\tau)$ and the dihedral angle $(\theta)$ between $\mathrm{S}_{2} \mathrm{CN}$ mean planes are closely comparable to values observed for the analogous features in most other tetrathiuram disulfides, as summarized in Fig. 3. Positive and negative values of $\tau$ occur with approximately equal frequency for tetra-
Figure 2

Stacking of tetraisobutylthiuram disulfide molecules along the $a$ axis of the unit cell, showing intermolecular $\mathrm{S} \cdots \mathrm{H}-\mathrm{C}$ close contacts. Displacement ellipsoids are represented at the $50 \%$ probability level. Parallel stacks fill in the $a b$ plane to form two-dimensional sheets, as shown. (Symmetry operations: $x+1, y, z ; x, y+1, z$.) 
Table 2

Experimental details.

\begin{tabular}{|c|c|}
\hline \multicolumn{2}{|l|}{ Crystal data } \\
\hline Chemical formula & $\mathrm{C}_{18} \mathrm{H}_{36} \mathrm{~N}_{2} \mathrm{~S}_{4}$ \\
\hline$M_{\mathrm{r}}$ & 408.73 \\
\hline Crystal system, space group & Triclinic, $P \overline{1}$ \\
\hline Temperature (K) & 100 \\
\hline$a, b, c(\AA)$ & $7.2449(11), 9.6102(14), 17.196(3)$ \\
\hline$\alpha, \beta, \gamma\left({ }^{\circ}\right)$ & $98.580(2), 94.540(2), 103.409(2)$ \\
\hline$V\left(\AA^{3}\right)$ & $1143.5(3)$ \\
\hline$Z$ & 2 \\
\hline Radiation type & Мо $K \alpha$ \\
\hline$\mu\left(\mathrm{mm}^{-1}\right)$ & 0.42 \\
\hline Crystal size (mm) & $0.17 \times 0.12 \times 0.06$ \\
\hline \multicolumn{2}{|l|}{ Data collection } \\
\hline Diffractometer & Bruker APEXII CCD \\
\hline Absorption correction & $\begin{array}{l}\text { Multi-scan (SADABS; Bruker, } \\
\text { 2016) }\end{array}$ \\
\hline$T_{\min }, T_{\max }$ & $0.745,0.977$ \\
\hline $\begin{array}{l}\text { No. of measured, independent and } \\
\text { observed }[I>2 \sigma(I)] \text { reflections }\end{array}$ & $17180,4168,3161$ \\
\hline$R_{\text {int }}$ & 0.057 \\
\hline$(\sin \theta / \lambda)_{\max }\left(\AA^{-1}\right)$ & 0.604 \\
\hline \multicolumn{2}{|l|}{ Refinement } \\
\hline$R\left[F^{2}>2 \sigma\left(F^{2}\right)\right], w R\left(F^{2}\right), S$ & $0.052,0.146,1.07$ \\
\hline No. of reflections & 4168 \\
\hline No. of parameters & 225 \\
\hline H-atom treatment & H-atom parameters constrained \\
\hline$\Delta \rho_{\max }, \Delta \rho_{\min }\left(\mathrm{e} \AA^{-3}\right)$ & $0.87,-0.35$ \\
\hline
\end{tabular}

Computer programs: APEX3 and SAINT (Bruker, 2016), SHELXT (Sheldrick, 2015a), SHELXL2014 (Sheldrick, 2015b) and SHELXTL (Sheldrick, 2008).

thium disulfides that have been characterized structurally by X-ray diffraction (Fig. 3). For those which do not reside on an inversion center (Kumar et al., 1990; Saczewski et al., 2006) or have conformations obviously perturbed by intermolecular interactions involving the pendant groups on nitrogen (Srinivasan et al., 2012), the average of the absolute value of $\tau$ is $88.4^{\circ}$, and the range is $78.0-99.0^{\circ}$. Similarly, the average value of $\theta$ is $86.1^{\circ}$, with a range of $79.0-90.0^{\circ}$.

\section{Synthesis and crystallization}

The synthesis procedure employed was that described by Kapanda et al., 2009. Pale-yellow block-shaped crystals of tetraisobutylthiuram disulfide (m.p. $343 \mathrm{~K}$ ) were obtained by slow evaporation of a $\mathrm{CH}_{2} \mathrm{Cl}_{2}$ solution. ${ }^{1} \mathrm{H} \mathrm{NMR}(\delta, \mathrm{ppm}$ in DMSO- $\left.d_{6}\right): 3.83\left[d, J=12 \mathrm{~Hz}, 8 \mathrm{H},-\mathrm{CH}_{2} \mathrm{CH}\left(\mathrm{CH}_{3}\right)_{2}\right], 2.39[\mathrm{br}$ $\left.m, 4 \mathrm{H},-\mathrm{CH}_{2} \mathrm{CH}\left(\mathrm{CH}_{3}\right)_{2}\right], 0.98\left[d, J=8 \mathrm{~Hz}, 12 \mathrm{H},-\mathrm{CH}_{2} \mathrm{CH}-\right.$ $\left.\left(\mathrm{CH}_{3}\right)_{2}\right], 0.87\left[d, J=8 \mathrm{~Hz}, 12 \mathrm{H},-\mathrm{CH}_{2} \mathrm{CH}\left(\mathrm{CH}_{3}\right)_{2}\right]$.

\section{Refinement details}

Crystal data, data collection and structure refinement details are summarized in Table 2. Hydrogen atoms were added in calculated positions and refined with isotropic displacement parameters that were approximately 1.2 times (for $-\mathrm{CH}$ - and $-\mathrm{CH}_{2}$ ) or 1.5 times (for $-\mathrm{CH}_{3}$ ) those of the carbon atoms to which they were attached. The $\mathrm{C}-\mathrm{H}$ distances assumed were $1.00,0.99$, and $0.98 \AA$ for the $-\mathrm{CH}-,-\mathrm{CH}_{2}$, and $-\mathrm{CH}_{3}$ types of hydrogen atoms, respectively.

\section{Funding information}

This work has been funded in part by support from the NSF (OIA 1539035 and DMR 1460637). The Louisiana Board of Regents is thanked for enhancement grant LEQSF-(200203)-ENH-TR-67 with which the Tulane $X$-ray diffractometer was purchased, and Tulane University is acknowledged for its ongoing support with operational costs for the diffraction facility.

\section{References}

Ajibade, P. A., Ejelonu, B. C. \& Omondi, B. (2012). Acta Cryst. E68, o2182.

Bai, F. Y., Li, X. T., Zhu, G. S. \& Xing, Y. H. (2010). Spectrochim. Acta Part A, 75, 1388-1393.

Bodige, S. G. \& Watson, W. H. (2003). Private communication (refcode: ULOXIC). CCDC, Cambridge, England.

Bruker (2016). APEX3, SADABS and SAINT. Madison, Wisconsin, USA.

Chieh, C. (1977). Can. J. Chem. 55, 1115-1119.

Chieh, C. (1978). Can. J. Chem. 56, 974-975.

Datta, R. N. \& Ingham, F. A. A. (2001). Rubber Additives Compounding Ingredients. In Rubber Technologist's Handbook, edited by S. K. De \& J. R. White, pp. 167-208. Shrewsbury, UK: Rapra Technology, Ltd.

Desiraju, G. R. \& Steiner, T. (1999). The Weak Hydrogen Bond in Structural Chemistry and Biology. Oxford University Press.

Dix, M. F. \& Rae, A. D. (1973). Cryst. Struct. Commun. 2, 159-162.

Fun, H.-K., Chantrapromma, S., Razak, I. A., Bei, F.-L., Jian, F.-F., Yang, X.-J., Lu, L. \& Wang, X. (2001). Acta Cryst. E57, o717-o718.

Groom, C. R., Bruno, I. J., Lightfoot, M. P. \& Ward, S. C. (2016). Acta Cryst. B72, 171-179.

Hall, V. J. \& Tiekink, E. R. T. (1995). Z. Kristallogr. 210, 701-702.

Heard, P. J. (2005). Prog. Inorg. Chem. 53, 1-70.

Hogarth, G. (2005). Prog. Inorg. Chem. 53, 71-561.

Horita, Y., Takii, T., Yagi, T., Ogawa, K., Fujiwara, N., Inagaki, E., Kremer, L., Sato, Y., Kuroishi, R., Lee, Y., Makino, T., Mizukami, H., Hasegawa, T., Yamamoto, R. \& Onozaki, K. (2012). Antimicrob. Agents Chemother. 56, 4140-4145.

Hu, S.-Z. (2000). Chin. J. Struct. Chem. 19, 234-238.

Ignatz-Hoover, F. \& To, B. H. (2016). Vulcanization. In Rubber Compounding: Chemistry and Applications, edited by B. Rodgers, ch. 11, pp. 461-522. Boca Raton, FL: CRC Press.

Ivanov, A. V., Zinkin, S. A., Forzling, W., Antzutkin, O. N. \& Kritikos, M. (2003). Koord. Khim. 29, 151-160.

Jian, F., Jiang, L., Fun, H.-K., Chinnakali, K., Razak, I. A. \& You, X. (1999). Acta Cryst. C55, 573-574.

Jiao, Y., Hannafon, B. N. \& Ding, W.-Q. (2016). Anticancer Agents Med. Chem. 16, 1378-1384.

Kapanda, C. N., Muccioli, C. G., Labar, G., Poupaert, J. H. \& Lambert, D. M. (2009). J. Med. Chem. 52, 7310-7314.

Karim, Md. M., Abser, Md. M., Hassan, M. R., Ghosh, N., Alt, H. G., Richards, I. \& Hogarth, G. (2012). Polyhedron, 42, 84-88.

Karle, I. L., Estlin, J. A. \& Britts, K. (1967). Acta Cryst. 22, 273-280.

Kumar, V., Aravamudan, G. \& Seshasayee, M. (1990). Acta Cryst. C46, 674-676.

Macrae, C. F., Bruno, I. J., Chisholm, J. A., Edgington, P. R., McCabe, P., Pidcock, E., Rodriguez-Monge, L., Taylor, R., van de Streek, J. \& Wood, P. A. (2008). J. Appl. Cryst. 41, 466-470.

Marøy, K. (1965). Acta Chem. Scand. 19, 1509.

Mutschler, J., Grosshans, M., Soyka, M. \& Rösner, S. (2016). Pharmacopsychiatry, 49, 137-141.

Nath, P., Bharty, M. K., Maiti, B., Bharti, B., Butcher, R. J., Wikaira, J. L. \& Singh, N. K. (2016). RSC Adv. 6, 93867-93880. 
Polyakova, I. N. \& Starikova, Z. A. (1990). Zh. Strukt. Khim. 31, 148152.

Prakasam, B. A., Ramalingam, K., Bocelli, G. \& Cantoni, A. (2009). Phosphorus Sulfur Silicon, 184, 2020-2033.

Quan, L., Yin, H., Zhai, J. \& Wang, D. (2008). Acta Cryst. E64, m108. Radwan, M. A. (1969). Forest Sci. 15, 439-445.

Raston, C. L. \& White, A. H. (1976). Aust. J. Chem. 29, 523-529.

Raya, I., Baba, I., Rosli, F. Z. \& Yamin, B. M. (2005). Acta Cryst. E61, o3131-03132.

Rout, G. C., Seshasayee, M. \& Aravamudan, G. (1982). Cryst. Struct. Commun. 11, 1389-1393.

Rowland, R. S. \& Taylor, R. (1996). J. Phys. Chem. 100, 7384-7391.

Saczewski, J., Frontera, A., Gdaniec, M., Brzozowski, Z., Sączewski, F., Tabin, P., Quiñonero, D. \& Deyà, P. M. (2006). Chem. Phys. Lett. 422, 234-239.

Saravanan, M., Prakasam, B. A., Ramalingam, K., Bocelli, G. \& Cantoni, A. (2005). Z. Anorg. Allg. Chem. 631, 1688-1692.

Sharma, V. K., Aulakh, J. S. \& Malik, A. K. (2003). J. Environ. Monit. 5, 717-723.

Sheldrick, G. M. (2008). Acta Cryst. A64, 112-122.

Sheldrick, G. M. (2015a). Acta Cryst. A71, 3-8.
Sheldrick, G. M. (2015b). Acta Cryst. C71, 3-8.

Shi, B. \& Wang, J. (1992). Xiamen Daxue Xuebao, Ziran Kexueban 31, 176-181.

Srinivasan, N., Thirumaran, S. \& Selvanayagam, S. (2012). Acta Cryst. E68, o3446.

Thirumaran, S., Ramalingam, K., Bocelli, G. \& Cantoni, A. (2000). Polyhedron, 19, 1279-1282.

Uludağ, N., Ateş, M., Çaylak Delibaş, N., Çelik, Ö. \& Hökelek, T. (2013). Acta Cryst. E69, o771.

Wang, Y. \& Liao, J. H. (1989). Acta Cryst. B45, 65-69.

Wang, Y., Liao, J.-H. \& Ueng, C.-H. (1986). Acta Cryst. C42, 1420 1423.

Williams, G. A., Statham, J. R. \& White, A. H. (1983). Aust. J. Chem. 36, 1371-1377.

Yamin, B. M., Suwandi, S. A., Fun, H.-K., Sivakumar, K. \& Shawkataly, O. B. (1996). Acta Cryst. C52, 951-953.

Ymén, I. (1983). Acta Chem. Scand. B, 37, 707-713.

Yu, B. \& Wang, J.-L. (2003). Qingdao Keji Daxue Xuebao, Ziran Kexueban 24, 394-397.

Zhai, J., Yin, H.-D., Li, F., Chen, S.-W. \& Wang, D.-Q. (2007). Acta Cryst. E63, o1969-o1970. 


\section{supporting information}

Acta Cryst. (2017). E73, 1764-1769 [https://doi.org/10.1107/S2056989017015158]

\section{Crystal structure of tetraisobutylthiuram disulfide}

\section{Patricia R. Fontenot, Bo Wang, Yueli Chen and James P. Donahue}

Computing details

Data collection: APEX3 (Bruker, 2016); cell refinement: SAINT (Bruker, 2016); data reduction: SAINT (Bruker, 2016); program(s) used to solve structure: SHELXT (Sheldrick, 2015a); program(s) used to refine structure: SHELXL2014 (Sheldrick, 2015b); molecular graphics: SHELXTL (Sheldrick, 2008); software used to prepare material for publication: SHELXTL (Sheldrick, 2008).

$N, N, N^{\prime}, N^{\prime}$-Tetrakis(2-methylpropyl)disulfane-1,2-dicarbothioamide

Crystal data

$\mathrm{C}_{18} \mathrm{H}_{36} \mathrm{~N}_{2} \mathrm{~S}_{4}$

$M_{r}=408.73$

Triclinic, $P \overline{1}$

$a=7.2449(11) \AA$

$b=9.6102(14) \AA$

$c=17.196(3) \AA$

$\alpha=98.580(2)^{\circ}$

$\beta=94.540(2)^{\circ}$

$\gamma=103.409(2)^{\circ}$

$V=1143.5(3) \AA^{3}$

\section{Data collection}

Bruker APEXII CCD

diffractometer

Radiation source: fine-focus sealed tube

Graphite monochromator

Detector resolution: 8.3333 pixels $\mathrm{mm}^{-1}$

$\varphi$ and $\omega$ scans

Absorption correction: multi-scan

(SADABS; Bruker, 2016)

$T_{\min }=0.745, T_{\max }=0.977$

Refinement

Refinement on $F^{2}$

Least-squares matrix: full

$R\left[F^{2}>2 \sigma\left(F^{2}\right)\right]=0.052$

$w R\left(F^{2}\right)=0.146$

$S=1.07$

4168 reflections

225 parameters

0 restraints
$Z=2$

$F(000)=444$

$D_{\mathrm{x}}=1.187 \mathrm{Mg} \mathrm{m}^{-3}$

Mo $K \alpha$ radiation, $\lambda=0.71073 \AA$

Cell parameters from 4848 reflections

$\theta=2.2-25.2^{\circ}$

$\mu=0.42 \mathrm{~mm}^{-1}$

$T=100 \mathrm{~K}$

Block, pale yellow

$0.17 \times 0.12 \times 0.06 \mathrm{~mm}$

17180 measured reflections

4168 independent reflections

3161 reflections with $I>2 \sigma(I)$

$R_{\text {int }}=0.057$

$\theta_{\text {max }}=25.4^{\circ}, \theta_{\min }=2.2^{\circ}$

$h=-8 \rightarrow 8$

$k=-11 \rightarrow 11$

$l=-20 \rightarrow 20$

Primary atom site location: structure-invariant direct methods

Hydrogen site location: inferred from neighbouring sites

$\mathrm{H}$-atom parameters constrained

$w=1 /\left[\sigma^{2}\left(F_{0}^{2}\right)+(0.0883 P)^{2}\right]$

where $P=\left(F_{\mathrm{o}}{ }^{2}+2 F_{\mathrm{c}}{ }^{2}\right) / 3$

$(\Delta / \sigma)_{\max }=0.001$

$\Delta \rho_{\max }=0.87 \mathrm{e} \AA^{-3}$

$\Delta \rho_{\min }=-0.35$ e $\AA^{-3}$ 


\section{Special details}

Experimental. The diffraction data were obtained from 3 sets of 400 frames, each of width $0.5^{\circ}$ in $\omega$, collected at $\varphi=$ $0.00,90.00$ and $180.00^{\circ}$ and 2 sets of 800 frames, each of width $0.45^{\circ}$ in $\varphi$, collected at $\omega=-30.00$ and $210.00^{\circ}$. The scan time was $60 \mathrm{sec} /$ frame.

Geometry. All esds (except the esd in the dihedral angle between two 1.s. planes) are estimated using the full covariance matrix. The cell esds are taken into account individually in the estimation of esds in distances, angles and torsion angles; correlations between esds in cell parameters are only used when they are defined by crystal symmetry. An approximate (isotropic) treatment of cell esds is used for estimating esds involving l.s. planes.

Fractional atomic coordinates and isotropic or equivalent isotropic displacement parameters $\left(\AA^{2}\right)$

\begin{tabular}{|c|c|c|c|c|}
\hline & $x$ & $y$ & $z$ & $U_{\text {iso }} * / U_{\text {eq }}$ \\
\hline S1 & $0.11097(10)$ & $0.22088(8)$ & $0.72835(4)$ & $0.0265(2)$ \\
\hline S2 & $0.35046(10)$ & $0.62092(8)$ & $0.68215(4)$ & $0.0268(2)$ \\
\hline S3 & $0.37169(10)$ & $0.47724(7)$ & $0.84025(4)$ & $0.0250(2)$ \\
\hline S4 & $0.13548(10)$ & $0.54581(8)$ & $0.82084(4)$ & $0.0253(2)$ \\
\hline N1 & $0.4760(3)$ & $0.2440(2)$ & $0.77920(12)$ & $0.0177(5)$ \\
\hline $\mathrm{N} 2$ & $0.0291(3)$ & $0.6939(2)$ & $0.71490(12)$ & $0.0184(5)$ \\
\hline $\mathrm{C} 1$ & $0.3217(4)$ & $0.2980(3)$ & $0.77839(15)$ & $0.0195(6)$ \\
\hline $\mathrm{C} 2$ & $0.1689(4)$ & $0.6296(3)$ & $0.73258(16)$ & $0.0208(6)$ \\
\hline $\mathrm{C} 3$ & $0.6574(4)$ & $0.3090(3)$ & $0.83096(15)$ & $0.0195(6)$ \\
\hline $\mathrm{H} 3 \mathrm{~A}$ & 0.6641 & 0.4125 & 0.8506 & $0.023^{*}$ \\
\hline H3B & 0.7650 & 0.3052 & 0.7993 & $0.023^{*}$ \\
\hline $\mathrm{C} 4$ & $0.6811(4)$ & $0.2335(3)$ & $0.90154(15)$ & $0.0253(6)$ \\
\hline $\mathrm{H} 4$ & 0.6567 & 0.1270 & 0.8813 & $0.030^{*}$ \\
\hline $\mathrm{C} 5$ & $0.8874(4)$ & $0.2875(4)$ & $0.94048(18)$ & $0.0375(8)$ \\
\hline $\mathrm{H} 5 \mathrm{~A}$ & 0.9155 & 0.3924 & 0.9597 & $0.056^{*}$ \\
\hline $\mathrm{H} 5 \mathrm{~B}$ & 0.9740 & 0.2674 & 0.9017 & $0.056^{*}$ \\
\hline $\mathrm{H} 5 \mathrm{C}$ & 0.9052 & 0.2375 & 0.9851 & $0.056^{*}$ \\
\hline C6 & $0.5418(5)$ & $0.2552(4)$ & $0.96030(17)$ & $0.0384(8)$ \\
\hline H6A & 0.5722 & 0.3576 & 0.9854 & $0.058^{*}$ \\
\hline H6B & 0.5511 & 0.1948 & 1.0009 & $0.058^{*}$ \\
\hline $\mathrm{H} 6 \mathrm{C}$ & 0.4115 & 0.2273 & 0.9328 & $0.058^{*}$ \\
\hline $\mathrm{C} 7$ & $0.4768(4)$ & $0.1089(3)$ & $0.72601(15)$ & $0.0215(6)$ \\
\hline H7A & 0.3433 & 0.0528 & 0.7085 & $0.026^{*}$ \\
\hline H7B & 0.5411 & 0.0491 & 0.7556 & $0.026^{*}$ \\
\hline $\mathrm{C} 8$ & $0.5774(4)$ & $0.1370(3)$ & $0.65392(16)$ & $0.0295(7)$ \\
\hline H8 & 0.7063 & 0.2031 & 0.6734 & $0.035^{*}$ \\
\hline C9 & $0.6088(4)$ & $-0.0045(3)$ & $0.61130(17)$ & $0.0303(7)$ \\
\hline H9A & 0.6814 & -0.0465 & 0.6480 & $0.045^{*}$ \\
\hline H9B & 0.6803 & 0.0145 & 0.5664 & $0.045^{*}$ \\
\hline $\mathrm{H} 9 \mathrm{C}$ & 0.4849 & -0.0727 & 0.5920 & $0.045^{*}$ \\
\hline $\mathrm{C} 10$ & $0.4765(5)$ & $0.2120(4)$ & $0.59941(19)$ & $0.0437(9)$ \\
\hline $\mathrm{H} 10 \mathrm{~A}$ & 0.5528 & 0.2336 & 0.5562 & $0.066^{*}$ \\
\hline H10B & 0.4598 & 0.3026 & 0.6291 & $0.066^{*}$ \\
\hline $\mathrm{H} 10 \mathrm{C}$ & 0.3510 & 0.1484 & 0.5775 & $0.066^{*}$ \\
\hline $\mathrm{C} 11$ & $-0.1242(4)$ & $0.7070(3)$ & $0.76450(15)$ & $0.0191(6)$ \\
\hline H11A & -0.1361 & 0.6320 & 0.7988 & $0.023^{*}$ \\
\hline
\end{tabular}




$\begin{array}{lllll}\text { H11B } & -0.2462 & 0.6864 & 0.7296 & 0.023^{*} \\ \text { C12 } & -0.0943(4) & 0.8561(3) & 0.81718(16) & 0.0266(7) \\ \text { H12 } & -0.1195 & 0.9262 & 0.7826 & 0.032^{*} \\ \text { C13 } & 0.1058(4) & 0.9157(3) & 0.86038(18) & 0.0337(7) \\ \text { H13A } & 0.1164 & 1.0126 & 0.8906 & 0.051^{*} \\ \text { H13B } & 0.1988 & 0.9220 & 0.8218 & 0.051^{*} \\ \text { H13C } & 0.1316 & 0.8510 & 0.8966 & 0.051^{*} \\ \text { C14 } & -0.2443(5) & 0.8413(3) & 0.87450(18) & 0.0353(7) \\ \text { H14A } & -0.2247 & 0.7710 & 0.9081 & 0.053^{*} \\ \text { H14B } & -0.3719 & 0.8077 & 0.8446 & 0.053^{*} \\ \text { H14C } & -0.2329 & 0.9358 & 0.9077 & 0.053^{*} \\ \text { C15 } & 0.0346(4) & 0.7671(3) & 0.64587(15) & 0.0209(6) \\ \text { H15A } & 0.1683 & 0.8198 & 0.6436 & 0.025^{*} \\ \text { H15B } & -0.0420 & 0.8400 & 0.6531 & 0.025^{*} \\ \text { C16 } & -0.0405(4) & 0.6652(3) & 0.56672(15) & 0.0232(6) \\ \text { H16 } & 0.0321 & 0.5883 & 0.5614 & 0.028^{*} \\ \text { C17 } & -0.2504(4) & 0.5917(4) & 0.56081(18) & 0.0360(8) \\ \text { H17A } & -0.3243 & 0.6653 & 0.5659 & 0.054^{*} \\ \text { H17B } & -0.2725 & 0.5343 & 0.6033 & 0.054^{*} \\ \text { H17C } & -0.2908 & 0.5276 & 0.5094 & 0.054^{*} \\ \text { C18 } & 0.0007(5) & 0.7523(3) & 0.50071(17) & 0.0385(8) \\ \text { H18A } & -0.0641 & 0.8315 & 0.5064 & 0.058^{*} \\ \text { H18B } & -0.0459 & 0.6888 & 0.4494 & 0.058^{*} \\ \text { H18C } & 0.1388 & 0.7927 & 0.5036 & 0.058^{*}\end{array}$

Atomic displacement parameters $\left(\AA^{2}\right)$

\begin{tabular}{lllllll}
\hline & $U^{11}$ & $U^{22}$ & $U^{33}$ & $U^{12}$ & $U^{13}$ & $U^{23}$ \\
\hline S1 & $0.0192(4)$ & $0.0294(4)$ & $0.0315(4)$ & $0.0091(3)$ & $0.0001(3)$ & $0.0041(3)$ \\
S2 & $0.0186(4)$ & $0.0343(4)$ & $0.0328(4)$ & $0.0133(3)$ & $0.0059(3)$ & $0.0101(3)$ \\
S3 & $0.0285(4)$ & $0.0215(4)$ & $0.0278(4)$ & $0.0155(3)$ & $-0.0030(3)$ & $0.0013(3)$ \\
S4 & $0.0285(4)$ & $0.0274(4)$ & $0.0283(4)$ & $0.0188(3)$ & $0.0074(3)$ & $0.0100(3)$ \\
N1 & $0.0191(12)$ & $0.0171(11)$ & $0.0176(11)$ & $0.0076(9)$ & $-0.0006(9)$ & $0.0013(9)$ \\
N2 & $0.0163(11)$ & $0.0180(11)$ & $0.0235(12)$ & $0.0087(9)$ & $0.0031(9)$ & $0.0045(9)$ \\
C1 & $0.0240(15)$ & $0.0182(13)$ & $0.0206(14)$ & $0.0106(11)$ & $0.0053(11)$ & $0.0070(11)$ \\
C2 & $0.0195(14)$ & $0.0196(14)$ & $0.0237(14)$ & $0.0061(11)$ & $0.0002(11)$ & $0.0037(11)$ \\
C3 & $0.0184(14)$ & $0.0165(13)$ & $0.0243(14)$ & $0.0066(11)$ & $-0.0010(11)$ & $0.0039(11)$ \\
C4 & $0.0280(16)$ & $0.0263(15)$ & $0.0239(15)$ & $0.0103(12)$ & $-0.0007(12)$ & $0.0075(12)$ \\
C5 & $0.0311(18)$ & $0.053(2)$ & $0.0319(17)$ & $0.0170(15)$ & $-0.0057(13)$ & $0.0111(15)$ \\
C6 & $0.0363(18)$ & $0.054(2)$ & $0.0283(17)$ & $0.0109(16)$ & $0.0034(14)$ & $0.0167(15)$ \\
C7 & $0.0260(15)$ & $0.0158(13)$ & $0.0255(15)$ & $0.0111(11)$ & $0.0052(12)$ & $0.0020(11)$ \\
C8 & $0.0365(18)$ & $0.0273(16)$ & $0.0288(16)$ & $0.0152(14)$ & $0.0092(13)$ & $0.0033(13)$ \\
C9 & $0.0390(18)$ & $0.0301(16)$ & $0.0289(16)$ & $0.0204(14)$ & $0.0079(13)$ & $0.0068(13)$ \\
C10 & $0.059(2)$ & $0.048(2)$ & $0.0371(19)$ & $0.0299(18)$ & $0.0191(17)$ & $0.0126(16)$ \\
C11 & $0.0152(13)$ & $0.0184(13)$ & $0.0267(14)$ & $0.0088(11)$ & $0.0041(11)$ & $0.0047(11)$ \\
C12 & $0.0342(17)$ & $0.0212(14)$ & $0.0301(16)$ & $0.0143(13)$ & $0.0071(13)$ & $0.0088(12)$ \\
C13 & $0.0413(19)$ & $0.0250(16)$ & $0.0339(17)$ & $0.0071(14)$ & $0.0042(14)$ & $0.0040(13)$ \\
C14 & $0.0410(19)$ & $0.0326(17)$ & $0.0377(18)$ & $0.0196(15)$ & $0.0118(15)$ & $0.0033(14)$
\end{tabular}




\begin{tabular}{lllllll} 
C15 & $0.0216(14)$ & $0.0179(13)$ & $0.0258(15)$ & $0.0101(11)$ & $0.0001(11)$ & $0.0050(11)$ \\
C16 & $0.0206(14)$ & $0.0256(14)$ & $0.0257(15)$ & $0.0102(12)$ & $0.0020(11)$ & $0.0048(12)$ \\
C17 & $0.0275(17)$ & $0.0418(19)$ & $0.0328(17)$ & $0.0045(14)$ & $0.0003(13)$ & $-0.0043(14)$ \\
C18 & $0.0384(19)$ & $0.045(2)$ & $0.0327(18)$ & $0.0081(15)$ & $0.0006(14)$ & $0.0130(15)$ \\
\hline
\end{tabular}

Geometric parameters $\left(\AA,{ }^{o}\right)$

\begin{tabular}{|c|c|c|c|}
\hline $\mathrm{S} 1-\mathrm{C} 1$ & $1.642(3)$ & C9-H9B & 0.9800 \\
\hline $\mathrm{S} 2-\mathrm{C} 2$ & $1.643(3)$ & $\mathrm{C} 9-\mathrm{H} 9 \mathrm{C}$ & 0.9800 \\
\hline $\mathrm{S} 3-\mathrm{C} 1$ & $1.826(3)$ & $\mathrm{C} 10-\mathrm{H} 10 \mathrm{~A}$ & 0.9800 \\
\hline $\mathrm{S} 3-\mathrm{S} 4$ & $1.9931(10)$ & $\mathrm{C} 10-\mathrm{H} 10 \mathrm{~B}$ & 0.9800 \\
\hline $\mathrm{S} 4-\mathrm{C} 2$ & $1.828(3)$ & $\mathrm{C} 10-\mathrm{H} 10 \mathrm{C}$ & 0.9800 \\
\hline $\mathrm{N} 1-\mathrm{C} 1$ & $1.337(3)$ & $\mathrm{C} 11-\mathrm{C} 12$ & $1.536(4)$ \\
\hline $\mathrm{N} 1-\mathrm{C} 7$ & $1.474(3)$ & $\mathrm{C} 11-\mathrm{H} 11 \mathrm{~A}$ & 0.9900 \\
\hline $\mathrm{N} 1-\mathrm{C} 3$ & $1.476(3)$ & $\mathrm{C} 11-\mathrm{H} 11 \mathrm{~B}$ & 0.9900 \\
\hline $\mathrm{N} 2-\mathrm{C} 2$ & $1.341(3)$ & $\mathrm{C} 12-\mathrm{C} 13$ & $1.516(4)$ \\
\hline $\mathrm{N} 2-\mathrm{C} 15$ & $1.466(3)$ & $\mathrm{C} 12-\mathrm{C} 14$ & $1.521(4)$ \\
\hline $\mathrm{N} 2-\mathrm{C} 11$ & $1.469(3)$ & $\mathrm{C} 12-\mathrm{H} 12$ & 1.0000 \\
\hline $\mathrm{C} 3-\mathrm{C} 4$ & $1.522(4)$ & $\mathrm{C} 13-\mathrm{H} 13 \mathrm{~A}$ & 0.9800 \\
\hline $\mathrm{C} 3-\mathrm{H} 3 \mathrm{~A}$ & 0.9900 & C13-H13B & 0.9800 \\
\hline $\mathrm{C} 3-\mathrm{H} 3 \mathrm{~B}$ & 0.9900 & $\mathrm{C} 13-\mathrm{H} 13 \mathrm{C}$ & 0.9800 \\
\hline $\mathrm{C} 4-\mathrm{C} 6$ & $1.510(4)$ & $\mathrm{C} 14-\mathrm{H} 14 \mathrm{~A}$ & 0.9800 \\
\hline $\mathrm{C} 4-\mathrm{C} 5$ & $1.527(4)$ & C14-H14B & 0.9800 \\
\hline $\mathrm{C} 4-\mathrm{H} 4$ & 1.0000 & $\mathrm{C} 14-\mathrm{H} 14 \mathrm{C}$ & 0.9800 \\
\hline C5-H5A & 0.9800 & $\mathrm{C} 15-\mathrm{C} 16$ & $1.532(4)$ \\
\hline C5-H5B & 0.9800 & $\mathrm{C} 15-\mathrm{H} 15 \mathrm{~A}$ & 0.9900 \\
\hline $\mathrm{C} 5-\mathrm{H} 5 \mathrm{C}$ & 0.9800 & C15-H15B & 0.9900 \\
\hline C6-H6A & 0.9800 & $\mathrm{C} 16-\mathrm{C} 17$ & $1.510(4)$ \\
\hline C6-H6B & 0.9800 & $\mathrm{C} 16-\mathrm{C} 18$ & $1.516(4)$ \\
\hline C6- $\mathrm{H} 6 \mathrm{C}$ & 0.9800 & $\mathrm{C} 16-\mathrm{H} 16$ & 1.0000 \\
\hline $\mathrm{C} 7-\mathrm{C} 8$ & $1.514(4)$ & C17-H17A & 0.9800 \\
\hline C7-H7A & 0.9900 & C17-H17B & 0.9800 \\
\hline C7-H7B & 0.9900 & $\mathrm{C} 17-\mathrm{H} 17 \mathrm{C}$ & 0.9800 \\
\hline $\mathrm{C} 8-\mathrm{C} 10$ & $1.506(4)$ & C18-H18A & 0.9800 \\
\hline $\mathrm{C} 8-\mathrm{C} 9$ & $1.520(4)$ & C18-H18B & 0.9800 \\
\hline $\mathrm{C} 8-\mathrm{H} 8$ & 1.0000 & $\mathrm{C} 18-\mathrm{H} 18 \mathrm{C}$ & 0.9800 \\
\hline C9-H9A & 0.9800 & & \\
\hline $\mathrm{C} 1-\mathrm{S} 3-\mathrm{S} 4$ & $104.71(9)$ & $\mathrm{H} 9 \mathrm{~B}-\mathrm{C} 9-\mathrm{H} 9 \mathrm{C}$ & 109.5 \\
\hline $\mathrm{C} 2-\mathrm{S} 4-\mathrm{S} 3$ & $104.22(9)$ & $\mathrm{C} 8-\mathrm{C} 10-\mathrm{H} 10 \mathrm{~A}$ & 109.5 \\
\hline $\mathrm{C} 1-\mathrm{N} 1-\mathrm{C} 7$ & $121.1(2)$ & $\mathrm{C} 8-\mathrm{C} 10-\mathrm{H} 10 \mathrm{~B}$ & 109.5 \\
\hline $\mathrm{C} 1-\mathrm{N} 1-\mathrm{C} 3$ & $125.3(2)$ & $\mathrm{H} 10 \mathrm{~A}-\mathrm{C} 10-\mathrm{H} 10 \mathrm{~B}$ & 109.5 \\
\hline $\mathrm{C} 7-\mathrm{N} 1-\mathrm{C} 3$ & $113.6(2)$ & $\mathrm{C} 8-\mathrm{C} 10-\mathrm{H} 10 \mathrm{C}$ & 109.5 \\
\hline $\mathrm{C} 2-\mathrm{N} 2-\mathrm{C} 15$ & $119.3(2)$ & $\mathrm{H} 10 \mathrm{~A}-\mathrm{C} 10-\mathrm{H} 10 \mathrm{C}$ & 109.5 \\
\hline $\mathrm{C} 2-\mathrm{N} 2-\mathrm{C} 11$ & $123.9(2)$ & $\mathrm{H} 10 \mathrm{~B}-\mathrm{C} 10-\mathrm{H} 10 \mathrm{C}$ & 109.5 \\
\hline $\mathrm{C} 15-\mathrm{N} 2-\mathrm{C} 11$ & $116.6(2)$ & $\mathrm{N} 2-\mathrm{C} 11-\mathrm{C} 12$ & $114.6(2)$ \\
\hline $\mathrm{N} 1-\mathrm{C} 1-\mathrm{S} 1$ & $126.7(2)$ & $\mathrm{N} 2-\mathrm{C} 11-\mathrm{H} 11 \mathrm{~A}$ & 108.6 \\
\hline $\mathrm{N} 1-\mathrm{C} 1-\mathrm{S} 3$ & $111.45(18)$ & $\mathrm{C} 12-\mathrm{C} 11-\mathrm{H} 11 \mathrm{~A}$ & 108.6 \\
\hline
\end{tabular}




\begin{tabular}{|c|c|c|c|}
\hline $\mathrm{S} 1-\mathrm{C} 1-\mathrm{S} 3$ & $121.80(15)$ & $\mathrm{N} 2-\mathrm{C} 11-\mathrm{H} 11 \mathrm{~B}$ & 108.6 \\
\hline $\mathrm{N} 2-\mathrm{C} 2-\mathrm{S} 2$ & $125.8(2)$ & $\mathrm{C} 12-\mathrm{C} 11-\mathrm{H} 11 \mathrm{~B}$ & 108.6 \\
\hline $\mathrm{N} 2-\mathrm{C} 2-\mathrm{S} 4$ & $112.26(19)$ & $\mathrm{H} 11 \mathrm{~A}-\mathrm{C} 11-\mathrm{H} 11 \mathrm{~B}$ & 107.6 \\
\hline $\mathrm{S} 2-\mathrm{C} 2-\mathrm{S} 4$ & $121.90(16)$ & $\mathrm{C} 13-\mathrm{C} 12-\mathrm{C} 14$ & $111.6(2)$ \\
\hline $\mathrm{N} 1-\mathrm{C} 3-\mathrm{C} 4$ & $113.4(2)$ & $\mathrm{C} 13-\mathrm{C} 12-\mathrm{C} 11$ & $113.9(2)$ \\
\hline $\mathrm{N} 1-\mathrm{C} 3-\mathrm{H} 3 \mathrm{~A}$ & 108.9 & $\mathrm{C} 14-\mathrm{C} 12-\mathrm{C} 11$ & $107.2(2)$ \\
\hline $\mathrm{C} 4-\mathrm{C} 3-\mathrm{H} 3 \mathrm{~A}$ & 108.9 & $\mathrm{C} 13-\mathrm{C} 12-\mathrm{H} 12$ & 108.0 \\
\hline $\mathrm{N} 1-\mathrm{C} 3-\mathrm{H} 3 \mathrm{~B}$ & 108.9 & $\mathrm{C} 14-\mathrm{C} 12-\mathrm{H} 12$ & 108.0 \\
\hline $\mathrm{C} 4-\mathrm{C} 3-\mathrm{H} 3 \mathrm{~B}$ & 108.9 & $\mathrm{C} 11-\mathrm{C} 12-\mathrm{H} 12$ & 108.0 \\
\hline $\mathrm{H} 3 \mathrm{~A}-\mathrm{C} 3-\mathrm{H} 3 \mathrm{~B}$ & 107.7 & $\mathrm{C} 12-\mathrm{C} 13-\mathrm{H} 13 \mathrm{~A}$ & 109.5 \\
\hline $\mathrm{C} 6-\mathrm{C} 4-\mathrm{C} 3$ & $112.5(2)$ & $\mathrm{C} 12-\mathrm{C} 13-\mathrm{H} 13 \mathrm{~B}$ & 109.5 \\
\hline $\mathrm{C} 6-\mathrm{C} 4-\mathrm{C} 5$ & $111.4(2)$ & $\mathrm{H} 13 \mathrm{~A}-\mathrm{C} 13-\mathrm{H} 13 \mathrm{~B}$ & 109.5 \\
\hline $\mathrm{C} 3-\mathrm{C} 4-\mathrm{C} 5$ & $108.8(2)$ & $\mathrm{C} 12-\mathrm{C} 13-\mathrm{H} 13 \mathrm{C}$ & 109.5 \\
\hline $\mathrm{C} 6-\mathrm{C} 4-\mathrm{H} 4$ & 108.0 & $\mathrm{H} 13 \mathrm{~A}-\mathrm{C} 13-\mathrm{H} 13 \mathrm{C}$ & 109.5 \\
\hline $\mathrm{C} 3-\mathrm{C} 4-\mathrm{H} 4$ & 108.0 & $\mathrm{H} 13 \mathrm{~B}-\mathrm{C} 13-\mathrm{H} 13 \mathrm{C}$ & 109.5 \\
\hline $\mathrm{C} 5-\mathrm{C} 4-\mathrm{H} 4$ & 108.0 & $\mathrm{C} 12-\mathrm{C} 14-\mathrm{H} 14 \mathrm{~A}$ & 109.5 \\
\hline $\mathrm{C} 4-\mathrm{C} 5-\mathrm{H} 5 \mathrm{~A}$ & 109.5 & $\mathrm{C} 12-\mathrm{C} 14-\mathrm{H} 14 \mathrm{~B}$ & 109.5 \\
\hline $\mathrm{C} 4-\mathrm{C} 5-\mathrm{H} 5 \mathrm{~B}$ & 109.5 & $\mathrm{H} 14 \mathrm{~A}-\mathrm{C} 14-\mathrm{H} 14 \mathrm{~B}$ & 109.5 \\
\hline $\mathrm{H} 5 \mathrm{~A}-\mathrm{C} 5-\mathrm{H} 5 \mathrm{~B}$ & 109.5 & $\mathrm{C} 12-\mathrm{C} 14-\mathrm{H} 14 \mathrm{C}$ & 109.5 \\
\hline $\mathrm{C} 4-\mathrm{C} 5-\mathrm{H} 5 \mathrm{C}$ & 109.5 & $\mathrm{H} 14 \mathrm{~A}-\mathrm{C} 14-\mathrm{H} 14 \mathrm{C}$ & 109.5 \\
\hline $\mathrm{H} 5 \mathrm{~A}-\mathrm{C} 5-\mathrm{H} 5 \mathrm{C}$ & 109.5 & $\mathrm{H} 14 \mathrm{~B}-\mathrm{C} 14-\mathrm{H} 14 \mathrm{C}$ & 109.5 \\
\hline $\mathrm{H} 5 \mathrm{~B}-\mathrm{C} 5-\mathrm{H} 5 \mathrm{C}$ & 109.5 & $\mathrm{~N} 2-\mathrm{C} 15-\mathrm{C} 16$ & $114.4(2)$ \\
\hline $\mathrm{C} 4-\mathrm{C} 6-\mathrm{H} 6 \mathrm{~A}$ & 109.5 & $\mathrm{~N} 2-\mathrm{C} 15-\mathrm{H} 15 \mathrm{~A}$ & 108.7 \\
\hline $\mathrm{C} 4-\mathrm{C} 6-\mathrm{H} 6 \mathrm{~B}$ & 109.5 & $\mathrm{C} 16-\mathrm{C} 15-\mathrm{H} 15 \mathrm{~A}$ & 108.7 \\
\hline $\mathrm{H} 6 \mathrm{~A}-\mathrm{C} 6-\mathrm{H} 6 \mathrm{~B}$ & 109.5 & $\mathrm{~N} 2-\mathrm{C} 15-\mathrm{H} 15 \mathrm{~B}$ & 108.7 \\
\hline $\mathrm{C} 4-\mathrm{C} 6-\mathrm{H} 6 \mathrm{C}$ & 109.5 & $\mathrm{C} 16-\mathrm{C} 15-\mathrm{H} 15 \mathrm{~B}$ & 108.7 \\
\hline $\mathrm{H} 6 \mathrm{~A}-\mathrm{C} 6-\mathrm{H} 6 \mathrm{C}$ & 109.5 & $\mathrm{H} 15 \mathrm{~A}-\mathrm{C} 15-\mathrm{H} 15 \mathrm{~B}$ & 107.6 \\
\hline $\mathrm{H} 6 \mathrm{~B}-\mathrm{C} 6-\mathrm{H} 6 \mathrm{C}$ & 109.5 & $\mathrm{C} 17-\mathrm{C} 16-\mathrm{C} 18$ & $111.2(2)$ \\
\hline $\mathrm{N} 1-\mathrm{C} 7-\mathrm{C} 8$ & $112.5(2)$ & $\mathrm{C} 17-\mathrm{C} 16-\mathrm{C} 15$ & $112.8(2)$ \\
\hline $\mathrm{N} 1-\mathrm{C} 7-\mathrm{H} 7 \mathrm{~A}$ & 109.1 & $\mathrm{C} 18-\mathrm{C} 16-\mathrm{C} 15$ & $108.2(2)$ \\
\hline $\mathrm{C} 8-\mathrm{C} 7-\mathrm{H} 7 \mathrm{~A}$ & 109.1 & $\mathrm{C} 17-\mathrm{C} 16-\mathrm{H} 16$ & 108.2 \\
\hline $\mathrm{N} 1-\mathrm{C} 7-\mathrm{H} 7 \mathrm{~B}$ & 109.1 & $\mathrm{C} 18-\mathrm{C} 16-\mathrm{H} 16$ & 108.2 \\
\hline $\mathrm{C} 8-\mathrm{C} 7-\mathrm{H} 7 \mathrm{~B}$ & 109.1 & $\mathrm{C} 15-\mathrm{C} 16-\mathrm{H} 16$ & 108.2 \\
\hline $\mathrm{H} 7 \mathrm{~A}-\mathrm{C} 7-\mathrm{H} 7 \mathrm{~B}$ & 107.8 & $\mathrm{C} 16-\mathrm{C} 17-\mathrm{H} 17 \mathrm{~A}$ & 109.5 \\
\hline $\mathrm{C} 10-\mathrm{C} 8-\mathrm{C} 7$ & $113.3(3)$ & $\mathrm{C} 16-\mathrm{C} 17-\mathrm{H} 17 \mathrm{~B}$ & 109.5 \\
\hline $\mathrm{C} 10-\mathrm{C} 8-\mathrm{C} 9$ & $112.2(3)$ & $\mathrm{H} 17 \mathrm{~A}-\mathrm{C} 17-\mathrm{H} 17 \mathrm{~B}$ & 109.5 \\
\hline $\mathrm{C} 7-\mathrm{C} 8-\mathrm{C} 9$ & $109.4(2)$ & $\mathrm{C} 16-\mathrm{C} 17-\mathrm{H} 17 \mathrm{C}$ & 109.5 \\
\hline $\mathrm{C} 10-\mathrm{C} 8-\mathrm{H} 8$ & 107.2 & $\mathrm{H} 17 \mathrm{~A}-\mathrm{C} 17-\mathrm{H} 17 \mathrm{C}$ & 109.5 \\
\hline $\mathrm{C} 7-\mathrm{C} 8-\mathrm{H} 8$ & 107.2 & $\mathrm{H} 17 \mathrm{~B}-\mathrm{C} 17-\mathrm{H} 17 \mathrm{C}$ & 109.5 \\
\hline $\mathrm{C} 9-\mathrm{C} 8-\mathrm{H} 8$ & 107.2 & $\mathrm{C} 16-\mathrm{C} 18-\mathrm{H} 18 \mathrm{~A}$ & 109.5 \\
\hline $\mathrm{C} 8-\mathrm{C} 9-\mathrm{H} 9 \mathrm{~A}$ & 109.5 & $\mathrm{C} 16-\mathrm{C} 18-\mathrm{H} 18 \mathrm{~B}$ & 109.5 \\
\hline $\mathrm{C} 8-\mathrm{C} 9-\mathrm{H} 9 \mathrm{~B}$ & 109.5 & $\mathrm{H} 18 \mathrm{~A}-\mathrm{C} 18-\mathrm{H} 18 \mathrm{~B}$ & 109.5 \\
\hline $\mathrm{H} 9 \mathrm{~A}-\mathrm{C} 9-\mathrm{H} 9 \mathrm{~B}$ & 109.5 & $\mathrm{C} 16-\mathrm{C} 18-\mathrm{H} 18 \mathrm{C}$ & 109.5 \\
\hline $\mathrm{C} 8-\mathrm{C} 9-\mathrm{H} 9 \mathrm{C}$ & 109.5 & $\mathrm{H} 18 \mathrm{~A}-\mathrm{C} 18-\mathrm{H} 18 \mathrm{C}$ & 109.5 \\
\hline $\mathrm{H} 9 \mathrm{~A}-\mathrm{C} 9-\mathrm{H} 9 \mathrm{C}$ & 109.5 & $\mathrm{H} 18 \mathrm{~B}-\mathrm{C} 18-\mathrm{H} 18 \mathrm{C}$ & 109.5 \\
\hline
\end{tabular}


supporting information

Hydrogen-bond geometry $\left(\AA,{ }^{\circ}\right)$

\begin{tabular}{lllll}
\hline$D-\mathrm{H} \cdots A$ & $D-\mathrm{H}$ & $\mathrm{H} \cdots A$ & $D \cdots A$ & $D-\mathrm{H} \cdots A$ \\
\hline $\mathrm{C} 3-\mathrm{H} 3 A \cdots \mathrm{S} 3$ & 0.99 & 2.34 & $2.907(3)$ & 115 \\
$\mathrm{C} 7-\mathrm{H} 7 A \cdots \mathrm{S} 1$ & 0.99 & 2.60 & $3.084(3)$ & 110 \\
$\mathrm{C} 8-\mathrm{H} 8 \cdots \mathrm{S} 1^{\mathrm{i}}$ & 1.00 & 2.97 & $3.834(3)$ & 146 \\
$\mathrm{C} 11-\mathrm{H} 11 A \cdots \mathrm{S} 4$ & 0.99 & 2.33 & $2.896(3)$ & 115 \\
$\mathrm{C} 11-\mathrm{H} 11 B \cdots \mathrm{S} 2 \mathrm{ii}$ & 0.99 & 2.87 & $3.810(3)$ & 160 \\
$\mathrm{C} 16-\mathrm{H} 16 \cdots \mathrm{S} 2$ & 1.00 & 2.91 & $3.473(3)$ & 117
\end{tabular}

Symmetry codes: (i) $x+1, y, z$; (ii) $x-1, y, z$. 